\title{
Electrodynamic Sorting of Industrial Scrap Metal ${ }^{\dagger}$
}

\author{
James R. Nagel, Dave Cohrs, Jacob Salgado and Raj K. Rajamani* \\ ${ }^{1}$ Department of Metallurgical Engineering, University of Utah, USA
}

\begin{abstract}
Electrodynamic sorting (EDX) is a new technology developed to sort industrial scrap metals. Under the present embodiment, an electromagnet is placed directly underneath a conveyor belt and then excited by an alternating electrical current to produce a time-varying magnetic field. As scrap particles pass through the field overhead, electrical eddy currents are induced throughout their volumes and then repelled away. If the frequency of excitation is very high (e.g., $12 \mathrm{kHz}$ ), then the lightweight aluminum particles tend to jump far more dramatically than heavier materials like copper, brass, and zinc. To demonstrate the principle, a small-scale prototype was assembled and tested. Using an 8-inch $(20 \mathrm{~cm})$ lane width, the system could process industrial scrap Zorba at a throughput of over $550 \mathrm{lbs} /$ hour $(225 \mathrm{~kg} / \mathrm{h})$ with an aluminum grade of $97.6 \%$ and a recovery of $93 \%$.
\end{abstract}

Keywords: eddy currents, recycling, scrap metal waste

\section{Introduction}

One of the greatest challenges in the metal recycling industry is the problem of sorting. Scrap metals rarely present in a clean, high-purity state, but instead tend to manifest as dirty mixtures of several distinct metals bundled together. Such contamination can dramatically reduce the economic value of an otherwise pure material, which means recyclers will often go to great lengths to separate metals down to their base constituents.

Depending on the specific material properties, some separation processes might be relatively straightforward to implement. Most notably, ferrous metals like iron and steel will strongly react to an applied magnetic field and can thus pull away from any other nonmagnetic materials nearby. Known simply as magnetic separation, the technology is typically embodied as a special array of permanent magnets, and it is a popular method for extracting ferromagnetic metals from shredded automobile waste (Oberteuffer, 1974; Svoboda and Fujita, 2003).

Another widespread technology is called eddy current separation (ECS), which utilizes a time-varying magnetic field to extract nonferrous metals from nonmetallic fluff. Typically, ECS is embodied as a mechanically rotating drum of permanent magnets, though other configurations are possible as well. A comprehensive historical review of

Received 28 May 2019; Accepted 8 July 2019

J-STAGE Advance published online 18 September 2019

1135 S 1460 E, ROOM 412, Salt Lake City, UT 84112-0102, USA

* Corresponding author: Raj K. Rajamani;

E-mail: raj.rajamani@utah.edu

TEL: +01-801-581-3107
ECS can be found in Smith et al. (2019) while Nagel (2018a) provides a detailed mathematical description of its underlying physics. In brief, the process works by inducing electrical eddy currents to flow throughout the interior of conductive particles as they pass through the timevarying magnetic field of the ECS. The relative motion between the electrical currents and the applied magnetic field then results in a net force of deflection that causes conductive particles to jump up and away from the applied field. In contrast, low conductivity materials (most notably, nonmetals) do not allow any significant eddy currents to flow throughout their interiors and thus experience no significant force of deflection.

Due to the widespread use of ECS among auto-shredding facilities, there is a tendency to produce relatively similar mixtures throughout the industry. In particular, one of the more common products is called Zorba, which is loosely defined as any combination of nonferrous scrap metals that is predominantly aluminum (ISRI, 2016). At present, Zorba is an especially difficult material to sort, and there does not yet appear to be any clear technological solution to the problem. For example, dense media separation attempts to exploit the disparity in mass density between so-called "light" metal fractions (e.g., aluminum and magnesium) and the "heavies" (e.g., copper, brass, and zinc) (Lee et al., 1994). Unfortunately, the process is prohibitively expensive to implement, and so very few recycling facilities are willing to invest in such an operation. Alternatively, hand sorting can be a viable method of separating Zorba, but only when the cost of human labor is very low (Taylor, 2017). It is also particularly difficult to sort the so-called "fine" materials with this 
an a or.ti

potet

clellin

One emerging technology that addresses such limitations is called electrodynamic sorting (EDX). The principle is similar to a traditional eddy current separator in that a time-varying magnetic field is used to induce eddy currents in scrap metal particles. However, rather than rotate a mechanical array of permanent magnets, the EDX system excites a fixed electromagnet with an alternating electrical current. This allows the frequency of excitation to reach far beyond the limits of a mechanical system, and it is capable of separating materials by both electrical conductivity and mass density.

In one EDX embodiment, Dholu et al. (2017) used a wire-wound, gapped toroid to generate a magnetic field at $6.5 \mathrm{kHz}$. The gap was then fed with $12.5 \mathrm{~mm}$ spheres of copper, aluminum, and brass, which were all sorted with perfect grade and recovery. The system was then operated at a frequency $1.9 \mathrm{kHz}$ to sort aluminum spheres by alloy composition. The results were likewise very impressive, with grade and recovery values reaching well-above $90 \%$. In another embodiment, Smith et al. (2017) used a similar gapped toroid at $21.4 \mathrm{kHz}$ to separate aluminum particles from silicon and CdTe as a simulation of shredded photovoltaic waste. Although the particles were much smaller $(1-3 \mathrm{~mm})$ and nonuniform, the grade and recovery values still reached above $95 \%$.

Despite the many advantages of EDX, the embodiment of a gapped toroid suffers from at least three limitations. The first limitation stems from the gap itself, which introduces a small bottleneck through which material must be fed. This bottleneck heavily restricts throughput, thereby preventing EDX from processing materials on the scale required by modern industrial recyclers (typically many tons per hour). A second limitation arises from the geometry of scrap material itself, which often manifests as thin, flaky particles. When fed into a gapped magnetic toroid, flat particles tend to orient themselves along the minimum cross-sectional area with respect to the applied magnetic field. This reduces the deflection forces experienced by such particles, thereby lowering the effectiveness of EDX as a sorting process. A third limitation arises when magnetic particles (e.g., iron and steel) accidentally enter the magnetic gap. While conductive, nonmagnetic particles repel away from the applied magnetic field, magnetic particles are instead pulled directly in. The result is thus a clogged gap that greatly impedes the performance of the sort.

To alleviate the limitations of a gapped toroid, this paper proposes the new EDX embodiment depicted in Fig. 1. Rather than drop material through a gap, the electromagnet core is instead oriented upright and then embedded directly underneath a conveyor belt. Doing so dramatically improves the throughput of the system, since material can now flow freely over the top of the magnet rather than funnel through a narrow bottleneck. It can also operate on thin, sheet-like particles, since gravity now forces them to orient along the direction of maximum cross-sectional exposure to the magnetic field. The embodiment is further robust against any accidental exposure to ferrous material because the presence of the conveyor belt naturally prevents particles from getting pulled into the gap.

\section{High throughput EDX}

As a demonstration of the new embodiment, the authors constructed the small-scale prototype depicted in Fig. 2. The system begins with a vibratory feeder that steadily pours scrap material onto a conveyor belt, after which it is rapidly accelerated up to a constant horizontal velocity of $2.0 \mathrm{~m} / \mathrm{s}$. Embedded directly within the conveyor assembly is an electromagnet core that is excited by an alternating electrical current. The tips of the electromagnet are placed in direct contact with the underside of the conveyor belt so that scrap particles pass directly through the magnetic field overhead. Depending on such factors as electrical conductivity, mass density, and physical geometry, some of the particles will repel up and away

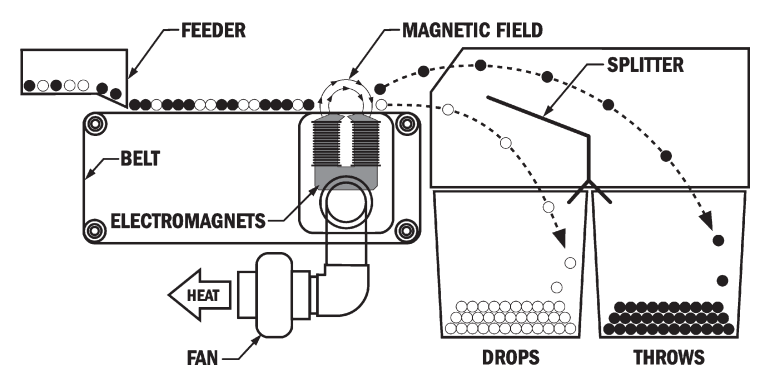

Fig. 1 Schematic diagram of the EDX embodiment with electromagnet cores placed directly underneath the conveyor belt.

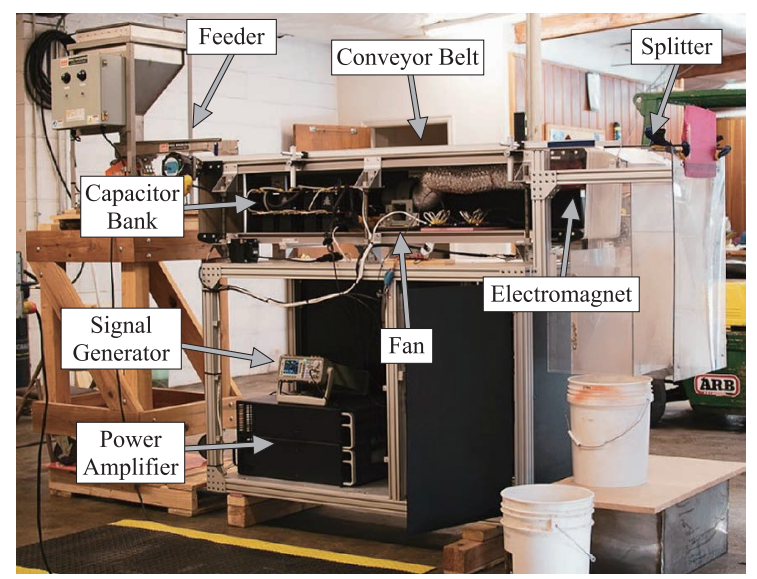

Fig. 2 Prototype assembly with major components indicated. 
from the magnet and are thus referred to as "throws." Likewise, any particles that pass through the field without any significant deflection are simply referred to as "drops." A mechanical splitter is then used to separate the throws from drops, which collect in their corresponding bins underneath. Finally, due to the excessive heat that builds up in the electromagnets, an axial flow fan is used to circulate air through the cores and maintain stable temperature.

Fig. 3 shows a close-up photograph of the electromagnet core that was embedded within the conveyor belt assembly. Since the cross-sectional shape is somewhat reminiscent of the letter $\mathrm{U}$, the authors chose to refer to it as a "U-core" geometry. At the top of the U are the tips, which were specially designed to funnel magnetic flux into a small volume of space overhead. The legs of the $U$ are then wrapped with insulated copper wire that is excited by an alternating electrical current of $7 A_{\mathrm{pk}}$ per coil. At the bottom of the $U$ is a base section to connect the legs together and stabilize the structure. The core length is then divided into three sub-sections, with each coil section electrically connected in parallel. Total core dimensions are roughly $20-\mathrm{cm}$ length, $8.6-\mathrm{cm}$ thickness, and 12-cm height.

The basic geometry of the U-core appears to have been conceived as far back as 1972 (Trondle, 1972), but seems to have only been used to remove nonferrous metals from nonmetallic minerals. Most likely, the earlier embodiments could not be excited at very high frequency or else the internal generation of eddy currents would rapidly heat the core beyond safe limits. For this reason, it is vital to choose a magnetic core material with both low conductivity and low hysteresis loss, and such materials may not have been commercially available so long ago. Fortunately, MnZn ferrites are now widely accessible, and they readily satisfy many of the desirable properties for an EDX core. Even so, the excessive drive current and magnetic field intensity still tends to generate a significant amount of heat throughout the core, and so it was necessary to circulate air with an axial flow fan in order to maintain stable temperatures.

In order to drive a sufficient electrical current through the EDX coils, it was necessary to utilize the circuit dia-

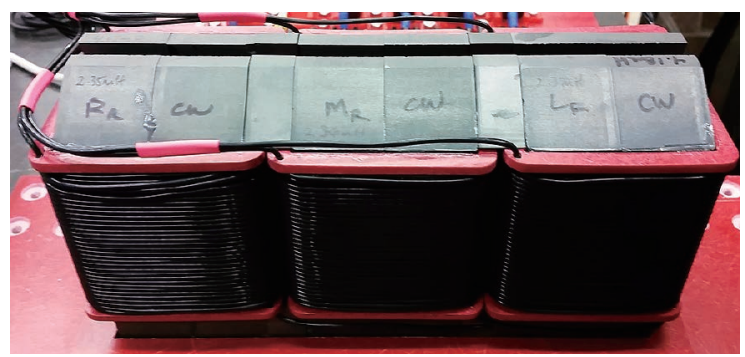

Fig. 3 Electromagnet core and windings. gram in Fig. 4. A signal generator begins by exciting the circuit with a sinusoidal voltage, which is then boosted by a high-power amplifier (20 V/V gain) to provide the necessary high-level drive current. The rest of the circuit then consisted of a series connection between some lumped capacitance $C$, a parallel connection of six inductors, and a small $0.1-\Omega$ resistor for current sensing.

The most noteworthy feature of the electrical circuit is the parallel connection between coils. Electrically speaking, each coil around the core may be treated as a large, lumped inductance with some equivalent series resistance (not shown). In principle, the coils could simply connect in series, but doing so would result in a total inductance of about $50 \mathrm{mH}$ or more. If we then imagine exciting the magnet at $7.0 A_{\mathrm{pk}}$ and a frequency of $f_{0}=12 \mathrm{kHz}$, the resulting voltage drop across the coils would exceed $26 \mathrm{kV}$. Since this is well beyond safe or practical, it was necessary to connect the coils in parallel, thereby reducing the impedance of the circuit. Specifically, if the current is divided among $N$ coils, then the total inductance of the circuit falls by a factor of $N^{2}$. Thus, when the circuit was connected into 6 parallel coils, the net inductance fell to a mere $1.3 \mathrm{mH}$. The trade-off to this approach is that total current must increase by a factor of 6 in order to maintain a consistent current density across the windings. Even so, when the coils were excited to a peak current of $42 A_{\mathrm{pk}}$, the coil voltage dropped to a manageable value of only $4.4 \mathrm{kV}$.

The final component of the circuit is capacitance, which must be chosen carefully to specify the desired resonant frequency. In particular, the resonant frequency of a series RLC circuit is given as

$$
f_{0}=\frac{1}{2 \pi \sqrt{L C}}
$$

Thus, to excite a $1.3 \mathrm{mH}$ inductance at a frequency of $12 \mathrm{kHz}$, the necessary capacitance evaluates to roughly $135 \mathrm{nF}$. In practice, however, there does not exist a single commercial device with such capacitance that can also withstand a full $42 A_{\mathrm{pk}}$ of current. The capacitance was therefore divided among multiple $10 \mathrm{nF}$ units that were

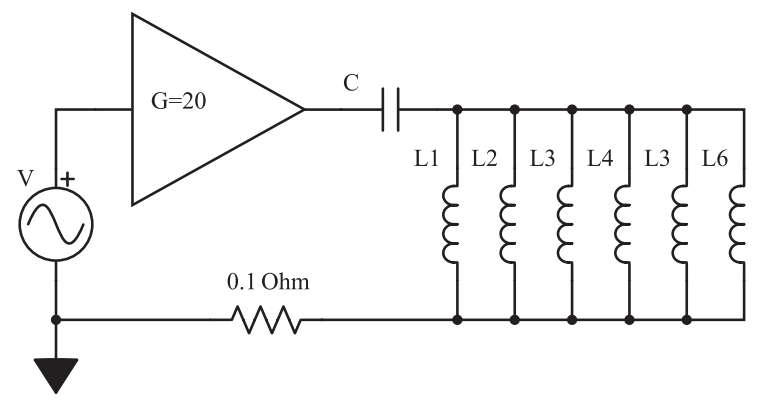

Fig. 4 Circuit diagram of the EDX drive electronics. 
lide wise connected in parallel (depicted as the single, luwitped capacitance $C$ in Fig. 4). Once assembled, the system was found to resonate at a frequency of $12.7 \mathrm{kHz}$.

\section{Separation theory}

The simplest mathematical model for a scrap metal particle is that of a sphere with radius $a$ and a uniform conductivity $\sigma$. The sphere is then excited by a timevarying magnetic field in sinusoidal steady state with angular frequency $\omega$ and a peak field intensity $B_{0}$ at the center. The magnetic field is also assumed to have a weak, linear gradient $\mathrm{d} B / \mathrm{d} x$ along the $x$-direction, resulting in a net, time-averaged force $F_{\text {avg }}$ that points along the direction of negative slope. Building on the work of Rony (1965) and Lohöfer (1989), we may calculate $F_{\text {avg }}$ using

$$
F_{\text {avg }}=-\frac{3 \pi B_{0} R^{3}}{\mu_{0}}\left[\frac{1}{3}-\frac{1}{q} \frac{\sinh (q)-\sin (q)}{\cosh (q)-\cos (q)}\right] \frac{\mathrm{d} B}{\mathrm{~d} x},
$$

where $q=\sqrt{2 \mu_{0} \sigma \omega a^{2}}$. Although Eqn. (2) is technically an approximate expression, Ray et al. (2018) measured the forces on metal spheres under magnetic excitation and showed it to be remarkably accurate.

Fig. 5 shows the calculated forces acting on $10 \mathrm{~mm}$ spheres with differing values of conductivity. In all cases, the applied magnetic field was set to $B_{0}=50 \mathrm{mT}$ with a field gradient of $\mathrm{d} B / \mathrm{d} x=1.0 \mathrm{~T} / \mathrm{m}$. The top graph shows $\sigma=60 \mathrm{MS} / \mathrm{m}$, which is typical for such metals as copper and silver. At the bottom end is $\sigma=10-20 \mathrm{MS} / \mathrm{m}$, which is typical for such metals as cast aluminum, brass, and zinc.

The key insight from this analysis is that, in the limit as $f \rightarrow \infty$, all forces tend towards the same, asymptotic value that is independent of conductivity. Acceleration, however, still depends on the overall mass of the particle,

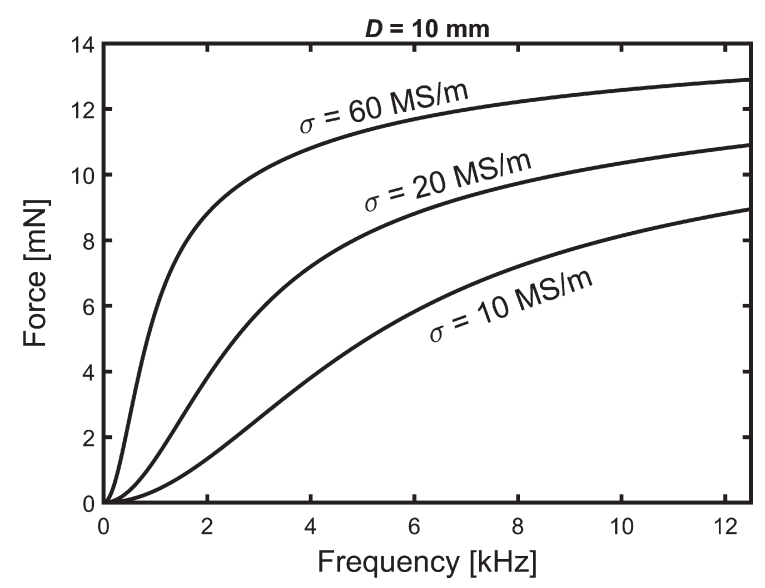

Fig. 5 Net force acting on a $10 \mathrm{~mm}$ conductive sphere as a function of excitation frequency. The peak magnetic field intensity is $B_{0}=50 \mathrm{mT}$ with a field gradient of $1.0 \mathrm{~T} / \mathrm{m}$. which is a function of mass density and physical geometry. For example, aluminum has a density of roughly $2.7 \mathrm{~g} / \mathrm{cm}^{3}$ while copper has a density of about $9.0 \mathrm{~g} / \mathrm{cm}^{3}$. The implication is that, with all other parameters held equally, a particle of aluminum will experience three times the acceleration of an equivalent copper particle. A high-frequency EDX assembly can thus be treated as a sophisticated density sorter.

Although the spherical model is very straightforward, there are many scrap particles that do not fit well into such a category. In particular, thin metal sheets are a common manifestation in any stream of fine Zorba, and it is necessary to apply complex numerical methods when calculating force and torque. Most notably, the work of Nagel (2018b) provides a simple finite-difference scheme that rapidly calculates of the eddy current density in thin rectangular sheets.

The most significant distinction between thin sheets and bulky spheres is that thin sheets throw far more dramatically after passing through the field of an EDX core. The reason is primarily due to the way in which metal sheets lie flat on the conveyor assembly, thereby exposing them to a much greater magnetic field intensity. The result is a much greater excitation of eddy currents and thus a much greater throw length. A practical consequence of this effect is that the new EDX embodiment can also sort particles by physical geometry as well as electrical conductivity and mass density.

\section{Test procedure}

To demonstrate the new EDX configuration, the authors acquired a $6000-1 \mathrm{bs}(2700 \mathrm{~kg})$ sample of fine Zorba from a local scrap recycling firm. Depicted in Fig. 6, the material was primarily distributed between $5-25 \mathrm{~mm}$ in size, thus earning it the nickname of "one inch minus." The simplest separation that can be performed by EDX is a removal of the light aluminum fraction from the heavies, which is very similar to what a dense-media plant would attempt to perform. To divide the labor into manageable chunks, the material was hand-shoveled into 5-gallon buckets and then processed one bucket at a time. Each bucket of material was weighed, sorted, and then weighed again after collecting into the throw/drop bins. Finally, after every tenth bucket, we extracted a small sample of material $(\approx 1 \mathrm{~kg})$ from the initial mixture, the throw pile, and the drop pile. All samples were then analyzed for metal composition using a portable XRF analyzer, which were then averaged to give the final composition. The entire procedure took about 3 days to complete.

It is important to note that the use of XRF analysis is different from a direct chemical analysis. The reason is because most common metals are actually complex alloys 
- Brass/Bronze: The most common alloys of copper are brass and bronze, which typically vary between 60-90\% copper content plus significant amounts of $\mathrm{Zn}$ and $\mathrm{Sn}$.

- Zinc/Other: Free zinc is the most common remaining metal in the heavy fraction. There also tends to be a small amount of other trace metals, such as $\mathrm{Cr}$, Sn, and stainless steel, but these are usually too insignificant to bother accounting for.

to calculate the resulting probabilities of throw and drop for the various metal compositions. The categories are thus summarized as:

- Al (Wrought/Cast): The most common component of Zorba is aluminum, which can take the form of either cast alloys or wrought alloys. Cast alloys typically contain about $8-10 \% \mathrm{Si}, 2-5 \% \mathrm{Cu}$, and $1-2 \%$ $\mathrm{Zn}$, while wrought alloys are typically comprised of about 96-98\% aluminum with a small percentage of other trace elements.

- Cu (Free): This category indicates any piece of relatively pure copper, such as copper pipe or copper wire, that is $96 \%$ copper content or more.
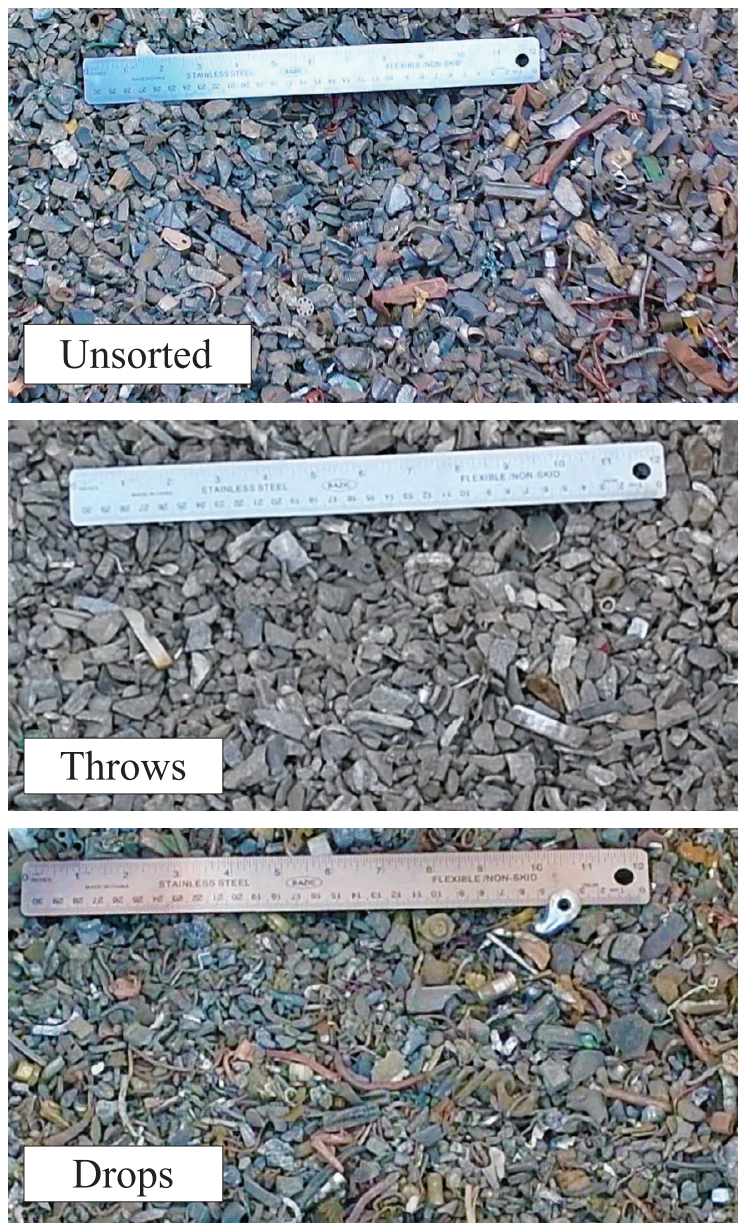

Fig. 6 (Top) Scrap metal feedstock consisting of fine Zorba donated by a local recycling firm. (Middle) Light aluminum fraction thrown by the EDX sorter. (Bottom) Heavy fraction in the drop bin.

\section{Results and discussion}

Fig. 6 provides photographs of the initial mixture, followed by typical throw and drop samples after sorting. Note that all images have been color-enhanced to help bring out the distinctly red and yellow hues of the copper and brass. Table 1 provides a summary of the XRF analysis for each category.

Over the course of the sort, it was found that throughput could vary quite significantly as a result of the vibratory feed rate and material composition. Although it could potentially reach as high as $800 \mathrm{lbs} / \mathrm{h}$, the materials tended to collide with each other around the divider and reduce grade/recovery. It was eventually decided that a feed rate of about $550 \mathrm{lbs} / \mathrm{h}(250 \mathrm{~kg} / \mathrm{h})$ was a practical throughput for the $20-\mathrm{cm}$ lane width.

The next metric of interest is power consumption, which was measured by probing the output voltage and drive current with an oscilloscope. Under normal operating conditions, the power amplifier would deliver a peak voltage of $33 \mathrm{~V}$ and a peak current of $36 \mathrm{~A}$. The net, time-averaged power consumption of the entire magnetic assembly was therefore about $600 \mathrm{~W}$. It is important to note, however, that a significant fraction of this power consumption appeared to be a result of coupling between the magnetic core and the metal frame around it. Although it is difficult to be sure how much power was wasted as a result, it was most likely in the range of about $100-200 \mathrm{~W}$. The implication is that, in principle, the magnetic core itself may only need to dissipate about $400 \mathrm{~W}$ during operation. After further accounting for the power consumption of the vibratory feeder $(\approx 400 \mathrm{~W})$, the

Table 1 Percent composition of Zorba before and after sorting.

\begin{tabular}{llll}
\hline Category & Pre-Sort & Throws & Drops \\
\hline Al (Wrought/Cast) & 71.4 & 97.6 & 15.0 \\
Copper (Free) & 4.4 & 0.5 & 12.7 \\
Brass/Bronze & 11.3 & 0.6 & 34.3 \\
Zn/Other & 12.9 & 1.3 & 38.0 \\
\hline Weight Percentage & 100 & 68.3 & 31.7 \\
\hline
\end{tabular}


gohiveytor drive motors $(\approx 200 \mathrm{~W})$, and a $50 \%$ efficiency within the power amplifier, the total power consumption for the entire EDX assembly reaches $1800 \mathrm{~W}$. Thus, given a feed rate of $250 \mathrm{~kg} / \mathrm{h}$, we arrive at $7.2 \mathrm{kWh}$ to sort one metric ton of material.

The final metrics of interest are aluminum grade in the throw bin and total aluminum recovery. Using the formal definitions of Dholu et al. (2017), the aluminum grade was found to reach an impressive $97.6 \%$. Of the small amount of contamination that also reached the throw bin, the majority appeared to result from thin sheet geometries. For example, US coins are a common product in shredded automobiles, and pennies in particular seemed to be the most prevalent. This resulted in a significant zinc contamination to the throws, since pennies are essentially thin disks of zinc with a thin copper coating.

To calculate the aluminum recovery, it is simply a matter of measuring the total aluminum mass in the throw bin and then dividing it by the original aluminum mass. The result was about $93 \%$ aluminum recovery from the original mixture, with the other $7 \%$ landing in the drop bin with the heavies. After examining the material, it was surmised that the majority of errors were due to small particle sizes (e.g., $5 \mathrm{~mm}$ or less), which could not throw effectively in the $12.7 \mathrm{kHz}$ field being applied. If the magnetic field were instead excited to much higher frequency, however (e.g., $20 \mathrm{kHz}$ or more), then is likely that recovery would have increased significantly as well.

On the other end of the size scale, many large particles of aluminum were simply too big to experience a proper throw by the magnet, but this can only be alleviated by redesigning the magnetic gap for a greater protrusion of field into the space above. Many other observed sources of error were also found to be purely kinematic in nature. For example, collisions between particles and the divider were common, and there is also a finite settling time for material as it drops onto the fast-moving conveyor belt. Fortunately, such errors can be corrected through revisions in the basic mechanical structure, which means future iterations are likely to yield even more reliable outcomes.

\section{Acknowledgments}

This work was funded by the United States Advanced Research Project Agency-Energy (ARPA-E) METALS Program under cooperative agreement grant DEAR0000411.

\section{Nomenclature}

$\begin{array}{ll}\text { ECS } & \text { Eddy Current Separator/Separation } \\ \text { EDX } & \text { Electrodynamic Sorting } \\ a & \text { Sphere Radius (m) } \\ B_{0} & \text { Magnetic Field Intensity (T) } \\ C & \text { Capacitance (F) } \\ f_{0} & \text { Resonant Frequency }(\mathrm{Hz}) \\ F & \text { Force (N) } \\ I & \text { Electric Current (A) } \\ L & \text { Inductance (H) } \\ \mu_{0} & \text { Vacuum Permeability (H/m) } \\ \sigma & \text { Electrical Conductivity (S/m) } \\ \omega & \text { Angular Frequency (rad/s) }\end{array}$

\section{References}

Dholu N., Nagel J.R., Cohrs D., Rajamani R.K., Eddy current separation of nonferrous metals using a variable-frequency electromagnet, KONA Powder and Particle Journal, 34 (2017) 241-247. DOI: 10.14356/kona.2017012

ISRI (Institute of Scrap Recycling Industries), Scrap specifications circular, Technical report, 2016. $<$ scrap2.org/specs/> accessed 22.08.2019.

Lee C.H., Cheau T.C., Chen S.T., Separating aluminum from shredded automotive scrap, Journal of The Minerals, Metals \& Materials Society (TMS), 46 (1994) 40-42. DOI: 10.1007/BF03220695

Lohöfer G., Theory of an electromagnetically levitated metal sphere I: Absorbed power, SIAM Journal of Applied Math, 49 (1989) 567-581. DOI: 10.1137/0149032

Nagel J.R., An analytic model for eddy current separation, Minerals Engineering, 127 (2018a) 277-285. DOI: 10.1016/ j.mineng.2018.08.025

Nagel J.R., Fast finite difference calculation of eddy currents in thin metal sheets, Applied Computational Electromagnetics Society Journal, 33 (2018b) 575-584.

Oberteuffer J.A., Magnetic separation: A review of principles, devices, and applications, IEEE Transactions on Magnetics, 10 (1974) 223-238. DOI: 10.1109/TMAG.1974.1058315

Ray J.D., Nagel J.R., Cohrs D., Rajamani R.K., Forces on particles in time-varying magnetic fields, KONA Powder and Particle Journal, 35 (2018) 251-257. DOI: 10.14356/kona.2018016

Rony P.R., The electromagnetic levitation of metals. in: Transactions of the Vacuum Metallurgy Conference 1964, American Vacuum Society, Boston, MA, 1965, pp.55-135.

Smith Y.R., Nagel J.R., Rajamani R.K., Electrodynamic eddy current separation of end-of-life PV materials, in: Lei Zhang et al. (Eds.) Energy Technology 2017: Carbon Dioxide Management and Other Technologies, Springer International Publishing, 2017, pp. 379-386. DOI: 10.1007/978-3- 
an $r$.

de $319-92192-3 \quad 37$

Smith Y.R., Nagel J.R., Rajamani R.K., Eddy current separation for recovery of non-ferrous metallic particles: A comprehensive review, Minerals Engineering, 133 (2019) 149-159. DOI: 10.1016/j.mineng.2018.12.025

Svoboda J., Fujita T., Recent developments in magnetic methods of material separation, Minerals Engineering, 16 (2003) 785-792. DOI: 10.1016/S0892-6875(03)00212-7
Taylor B., Absorbing the zorba. Recycling Today, (2017) $<$ recyclingtoday.com/article/absorbing-the-zorba/> accessed 22.08.2019.

Trondle H.M., Process for the separation of electrically conductive or semiconducting mineral particles of electrically non-conductive mineral particles and device for carrying out this process, German Patent (1972) DE2059166A1.

\section{Authors' Short Biographies}
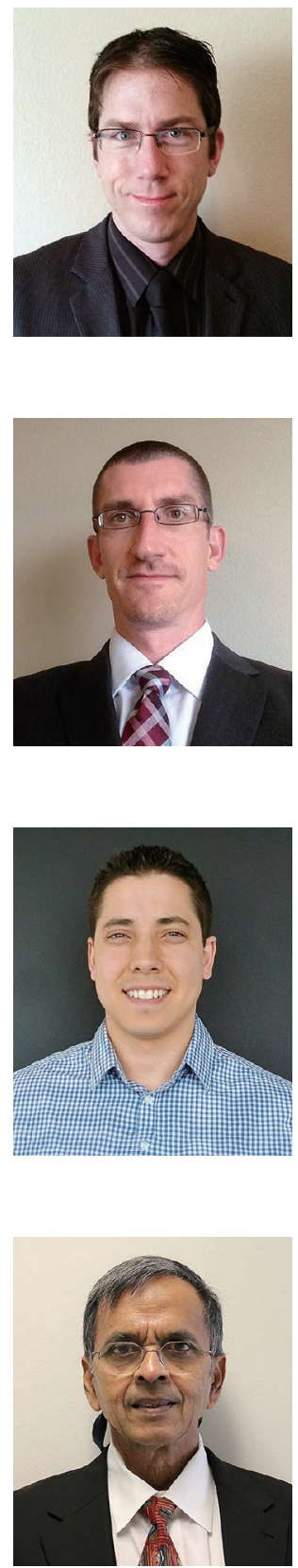

James Nagel

James Nagel completed his PhD in 2011 from the University of Utah in Salt Lake City, Utah, where he was awarded the Stockham Medal of Excellence for conspicuously effective teaching. His background is in electrical engineering with a specialty in applied electromagnetics and numerical methods. He now works as a research associate for the University of Utah and is a co-founder for EDX Magnetics, LLC.

\section{Dave Cohrs}

Dave Cohrs completed his Bachelor of Science in 2007 at the University of Maryland in Baltimore, Maryland, where he studied Environmental Science with an emphasis on Analytical Chemistry. He managed water quality laboratories at public aquariums for 16 years and has consulted for aquariums, zoos, and academia on water chemistry and life support topics. He now works for the University of Utah as a research associate and is a co-founder for EDX Magnetics, LLC.

\section{Jacob Salgado}

Jacob Salgado completed his Bachelor of Science in Metallurgical Engineering from the University of Utah in 2018. He is a decorated military veteran who served in the United States Army from 2010-2013 and deployed overseas in support of Operation Enduring Freedom. He has been working as a research associate at the University of Utah since 2015, and he has also worked as a product engineer for EDX Magnetics, LLC, since 2018.

\section{Raj Rajamani}

Raj Rajamani has been on the faculty of the Metallurgical Engineering Department of the University of Utah, Salt Lake City, Utah, USA since 1980. Currently he holds the position of Professor. His research interests include population balance modeling of tumbling mills, computational fluid dynamics of hydrocyclones, discrete element modeling of semi-autogenous grinding mills, eddy current sorting of metallic particles and modeling of high pressure grinding rolls. He received the Antoine M. Gaudin Award, presented by the Society of Mining, Metallurgy and Exploration Engineers Inc. in the year 2009. 